\title{
Analysis of central macular thickness and choroidal thickness changes in patients with cardiovascular risk factors
}

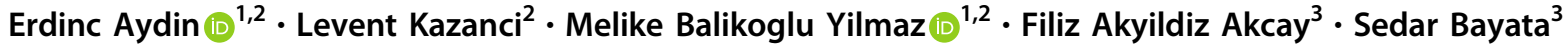

Received: 5 January 2019 / Revised: 7 January 2020 / Accepted: 16 January 2020 / Published online: 28 January 2020

(c) The Author(s), under exclusive licence to The Royal College of Ophthalmologists 2020

\begin{abstract}
Objectives The aim of this study was to evaluate central macular thickness (CMT) and choroidal thickness (CT) in the eyes of patients with cardiovascular risk factors (CVRF).

Methods A cross-sectional, prospective observational study of 92 patients with CVRF and 21 healthy individuals was conducted. Patients were divided into four groups according to the SCORE system. CMT was evaluated via spectraldomain-optical coherence tomography (SD-OCT). CT at five defined points (subfoveal) [SF] and nasal $500 \mu \mathrm{m}$ [N0.5] and $1500 \mu \mathrm{m}$ [N1.5] and $500 \mu \mathrm{m}$ [T0.5] and temporal $1500 \mu \mathrm{m}$ [T1.5] from the center of the fovea were measured via enhanced depth imaging (EDI)-OCT.

Results Mean SFCT at right eyes (RE) and left eyes (LE) were $311.21 \pm 77.7 \mu \mathrm{m}$ and $303.5 \pm 49.6 \mu \mathrm{m}$, respectively, in patients with mild CVRF (Group 1); $266.5 \pm 63.2 \mu \mathrm{m}$ and $267.0 \pm 62.6 \mu \mathrm{m}$, respectively, in patients with moderate CVRF (Group 2); $264.7 \pm 57.5 \mu \mathrm{m}$ and $272.3 \pm 64.6 \mu \mathrm{m}$, respectively, in patients with high CVRF (Group 3); $272.3 \pm 64.6 \mu \mathrm{m}$ and $271.2 \pm 63.4 \mu \mathrm{m}$, respectively, in patients with very high-risk CVRF (with coronary arterial disease (CAD) (Group 4); and $352.0 \pm 74.4 \mu \mathrm{m}$ and $363.1 \pm 89.0 \mu \mathrm{m}$, respectively, in the control group. CT (at both eyes) was significantly lower at the subfoveal location in all study groups $(P<0.05)$, but at nasal and at temporal quadrants of group 3 and group $4(P<0.05)$. No significant difference in CMT was detected between the study and control groups.

Conclusions This study demonstrated that CVRF might result in a remarkably thinner CT. Furthermore, subretinal drusenoid deposits were detected at a higher rate in the patients with CVRF than controls, and that rate increased in accordance with the severity of CAD. In the future, changes in CT may be used as a promising novel biomarker as part of the SCORE system prior to the development of CAD.
\end{abstract}

\section{Introduction}

Coronary heart disease (CHD), also known as coronary artery disease (CAD), is a major cause of death worldwide and occurs 2- to 5-times more frequently in men than in women of middle-age. Certain risk factors, such as elevated low-density lipoprotein (LDL) cholesterol, age, smoking,

Erdinc Aydin

erdincaydin@yahoo.com

1 Department of Ophthalmology, Izmir Katip Celebi University, Faculty of Medicine, Izmir, Turkey

2 Eye Clinic, Izmir Katip Celebi University, Ataturk Training and Research Hospital, Izmir, Turkey

3 Cardiology Clinic, Izmir Katip Celebi University, Ataturk Training and Research Hospital, Izmir, Turkey diabetes mellitus (DM), hypertension, kidney disease, are used clinically to identify susceptible individuals [1-3].

Changes in retinal vessels may reflect coronary microvascular damage. Computerized techniques have been developed that measure retinal vessels via retinal imaging, which has enabled the anticipation of increased risk of CAD in epidemiological studies $[4,5]$.

The main function of the choroid is to supply blood to the outer one-third of the neuroretina and the retinal pigment epithelium (RPE), which provides oxygen and nutrients to the fovea. Choroid vessels are controlled neurogenically, unlike the retinal vascular network. Enhanced depth imaging optical coherence tomography [EDI-OCT], as defined by Spaide, enables in vivo crosssectional imaging of the choroid [6].

The thickness of healthy choroid changes between 250 and $400 \mu \mathrm{m}$ subfoveal and becomes thinner in the temporal and nasal regions [7,8]. The relationship between the 
choroid and cardiovascular system as a biomarker of cardiovascular disease (CVD) may be beneficial for identifying patients at increased risk of retinal disease. Choroidal thickness (CT) varies with age, gender, diurnal, axial length, pregnancy, and various systemic and ocular diseases [9].

The choroid may be prone to arteriosclerotic processes to the same degree as other organs. Severe hypertensive retinopathy is related to hypertensive choroidopathy and choroidal thickening [10]. Hypercholesterolemia is associated with choroidal thickening [11], whereas cigarette smoking [3], ocular ischemic syndrome [12], chronic heart failure [13], and systemic hypertension [14] are associated with a thinner choroid. On the other hand, there are conflicting reports regarding the association of carotid artery stenosis [15] and DM [16] with CT. Choroidal abnormalities can occur in several posterior segment diseases, including polypoidal choroidal vasculopathy and agerelated macular degeneration (AMD). AMD, which is associated with primary lesions of soft drusen, is a leading cause of blindness in its advanced forms of geographic atrophy and choroidal neovascularization [17]. Reticular macular disease (RMD) [18], which is associated with lipidrich subretinal drusenoid deposits (SDD) [19], has been recently recognized, as the most aggressive form of intermediate AMD (iAMD), doubling the risk of soft drusen progressing to advanced AMD, both exudative and nonexudative [20, 21]. SDD is routinely accompanied by choroidal vascular abnormalities, including choroidal thinning and/or insufficiency of the choriocapillaris (CC) $[22,23]$. SDD and reticular pseudodrusen (RPD) with choroidal thinning constitute RMD, which has been found in patients with CAD [24] and other systemic vascular diseases, including renal insufficiency [25], hypertension, and angina [26]. These observations have led to the theory that CAD may, in fact, drive this severe form of AMD.

The aim of our study was to investigate central macular thickness (CMT) and CT in the eyes of patients with cardiovascular risk factors or CAD. Hence, it is possible to detect the potential association between $\mathrm{CT}$ and patients that are candidates for CVD.

\section{Material and methods}

\section{Study population and design}

This cross-sectional, prospective observational study was conducted with patients and healthy subjects recruited from Izmir Katip Celebi University, Ataturk Training and Research Hospital's Cardiology and Eye Clinics, from August 2017 to September 2018. This study adhered to the tenets of the Declaration of Helsinki and was approved by the local ethics committee (approval number:
81-10082017). Informed consent was obtained from all participants.

Patients with CVR factors were divided into four groups (Group 1: 28 patients with mild cardiovascular risk; Group 2: 30 patients with moderate cardiovascular risk; Group 3: 10 patients with high cardiovascular risk; Group 4: 24 patients with CAD were defined as the patients with very high cardiovascular risk that undergoing follow-up at the Cardiology Clinic. The 21 controls were similarly formed by age and gender-matched healthy subjects, who had applied to the Eye Clinic of the same hospital for examination. Diagnosis of $\mathrm{CAD}$ and cardiovascular risk assessment of patients were performed by cardiologists based on the SCORE (Systematic Coronary Risk Estimation) system, which estimates the 10year risk of fatal cardiovascular disease [27].

Inclusion criteria for patients with CAD included previous acute myocardial infarction (AMI), acute coronary syndrome or coronary revascularization (with a stent or coronary artery bypass graft), or significant plaque on coronary angiography. CVRF of patients were defined by the SCORE system [27].

Exclusion criteria for patients included high myopia, amblyopia, and advanced cataracts; or a history of retinal vascular disease, retinal dystrophy, glaucoma, history of uveitis, and age-related macular degeneration (AMD) retinal surgery; or laser photocoagulation systemic or ocular disease that prevented examination of the cornea and retina. Patients that had used a vasoconstrictive drug within the last three months were omitted from both the patient and control groups. Patients with a refractive error that was less than -3 diopters (D) or more than $+3 \mathrm{D}$ and a best-corrected visual acuity (BCVA) of 20/25 or better and any associated systematic disorders that may affect the eyes (e.g., uncontrolled diabetes, usage of systemic corticosteroids, or hypertension) were included.

Participants underwent a full ophthalmologic examination to determine visual acuity, spherical equivalent (SE) values of refractive errors, intraocular pressures (IOP), and choroidal thickness. Choroidal thickness was measured using Cirrus high-definition optical coherence tomography (HD-OCT, Cirrus Version 6.0; Carl Zeiss Meditec, Dublin, CA, USA).

All subjects underwent a complete physical examination. Blood parameters included a complete blood count and clinical chemistry (glucose, sodium, potassium, creatinine, uric acid, total cholesterol, triglycerides, alanine aminotransferase, aspartate aminotransferase, gamma-glutamyl transferase, total bilirubin, and total protein).

All HD-OCT scans were performed by the same experienced technician during the day (03:00-04:00 pm) after pupil dilation. Each participant's head was fixed in the restraint while five radial retinal scans were performed in which the eye-without blinking or moving-remained focused on the international target. Only complete and 
well-centered scans that had a signal strength of at least six and contained no motion or blinking artifacts were accepted. After each examination, two independent masked graders evaluated the best image on a computer screen. The image was taken and used for analysis when the two graders determined that both the inner and outer borders of the choroid were distinguishable. The choroidal image was obtained according to the protocol, which was centered foveally and spaced at $0.25 \mathrm{~mm}$.

The HD 5 Line Raster protocol consists of $6.0 \mathrm{~mm}$ parallel lines with 1024 A-scans/B-scans, with an average of four B-scans per image. Cirrus software was used to avoid inversion of the image, which results in pixelation, and lowresolution Cirrus software allowed us to bring the choroid into closer proximity to the zero-delay line. An image of the macula's thinnest point was selected to prevent the effect of positioning on the measured thickness of the fovea. The image size was doubled, foveally-centered, and the subfoveal choroidal thickness (SFCT) from the outer portion of the hyper-reflective line corresponding to RPE and to the inner surface of the sclera, using the linear measurement tool of the Cirrus software. The thicknesses of the choroid at the areas of $500 \mu \mathrm{m}$ (N0.5) and $1500 \mu \mathrm{m}$ (N1.5) nasal and $500 \mu \mathrm{m}$ (T0.5) and $1500 \mu \mathrm{m}$ (T1.5) temporal to the fovea were examined in all groups (Fig. 1a-e). Two independent raters measured the images without any information of the eye or other observer results, as described previously, and the average of the two measurements was calculated. Differences between the readings of the masked physicians were within $10 \%$ of the mean.

\section{Statistical analysis}

Statistical analysis was performed using the statistical package SPSS v. 18.0 (SPSS Inc., IBM Corp, Chicago, Illinois, USA). For general statistical reporting, the mean value of each data set was calculated along with the standard deviation (SD). Normality for each continuous variable for both groups was evaluated using the Kolmogorov-Smirnov test, histograms, and p-p plots. Differences in measured parameters between the two groups were analyzed by Student's $t$ test and chi-square test. Correlations between variables were analyzed with Pearson's or Spearman's correlation coefficients. A $p$ value of 0.05 was considered to be statistically significant.

\section{Results}

The mean ages of the study groups were as follows: $40.1 \pm 6.5$ years (range 29-56 years) for Group $1(n=$ $24) ; 56.9 \pm 9.3$ years (range 38-76 years) for Group 2 $(n=30) ; 68.0 \pm 5.2$ years (range $59-75$ years) for Group $3(n=10) ; 59.0 \pm 9.9$ years (range $42-78$ years) for Group $4(n=24)$; and $48.8 \pm 5.9$ years (range $40-62$ years) for the control group $(n=21)$.

Age and gender distributions were significantly different between the groups $(p=0.032$ and $p=0.021)$. Characteristics of the study and control groups are shown in Table 1.

EDI-OCT measurement and mean SFCT (at right and left eyes) were $311.21 \pm 77.7 \mu \mathrm{m}$ and $303.5 \pm 49.6 \mu \mathrm{m}$, respectively, in patients with mild CVRF (Group 1); $266.5 \pm 63.2$ $\mu \mathrm{m}$ and $267.0 \pm 62.6 \mu \mathrm{m}$, respectively, in patients with moderate CVRF (Group 2); $264.7 \pm 57.5 \mu \mathrm{m}$ and $272.3 \pm$ $64.6 \mu \mathrm{m}$, respectively, in patients with severe CVRF (Group 3); $272.3 \pm 64.6 \mu \mathrm{m}$ and $271.2 \pm 63.4 \mu \mathrm{m}$, respectively, in patients with CAD (Group 4); and 352.0 $\pm 74.4 \mu \mathrm{m}$ and $363.1 \pm 89.0 \mu \mathrm{m}$, respectively, in the control group.

The mean SFCT for all study groups was lower than those in controls for both eyes and these differences were statistically significant (Group 1: right eye $(\mathrm{R}) p=0.075$, left eye (L) $p=0.014$; Group 2: (R) $p=0.0001$, (L) $p=0.0001$; Group 3: (R) $p=0.001(\mathrm{~L}), p=0.001$; and Group $4(\mathrm{R}) p=$ 0.0001 (L) $p=0.0001$; independent $t$ test) (Fig. 1a-d).

At each nasal and temporal determination, the mean CT values of both eyes of patients (Group 2, Group 3, and Group 4) were significantly decreased when compared with controls $((\mathrm{R} / \mathrm{L})$ at N0.5: G2 $p=0.007$ and $p=0.002$, respectively; G3: $p=0.003$ and $p=0.039$, respectively; G4: $p=0.004$ and $p=0.01$, respectively. For N1.5, G2 $p=0.0001$ and $p=0.0001$, respectively; G3: $p=0.004$ and $p=0.028$, respectively; G4 $p=0.003$ and $p=0.007$, respectively. For T0.5, G2 $p=0.001$ and $p=0.004$, respectively; G3 $p=0.005$ and $p=0.015$, respectively; G4 $p=0.008$ and $p=0.015$, respectively. For T1.5: G2 $p=$ 0.003 and $p=0.002$, respectively; G3 $p=0.027$ and $p=$ 0.023 , respectively; G4 $p=0.036$ and $p=0.004$, respectively; independent $t$ test) (Table 2) (Fig. 1a-e).

No significant differences in the CMT of the right (RE) and the left eyes (LE) between the study and control groups were detected (RE: $p=0.057$, LE: $p=0.097$ ).

For the study and control groups, SFCT, CT (T.0.5, T.1.5, N0.5, and N1.5), and CMT were found to be inversely correlated with age. LDL levels negatively correlated with SFCT in both eyes.

In our study, the ratio of patients with high cholesterol (Ch) and low LDL level were: 7/24 (29.1\%) and 9/24 (37.5\%), respectively, in Group 1; 23/30 (76.6\%) and 2/30 (6.6\%), respectively, in Group 2; 7/10 (70\%) and 2/10 (20\%), respectively, in Group 3; 7/24 (29.1\%) and 13/24 $(54.1 \%)$, respectively, in Group 4.

We also explored the rate of SDD in each of the study groups and controls found $35.7 \%$ (stage 1) in Group 1, $46.6 \%$ (stage 1) in Group 2, 40\% (stage 1) in Group 3 and $70.8 \%$ (Stage 1: $12.5 \%$ and Stage 2: $58.3 \%$ ) in Group 4 and $23.8 \%$ in control group. 


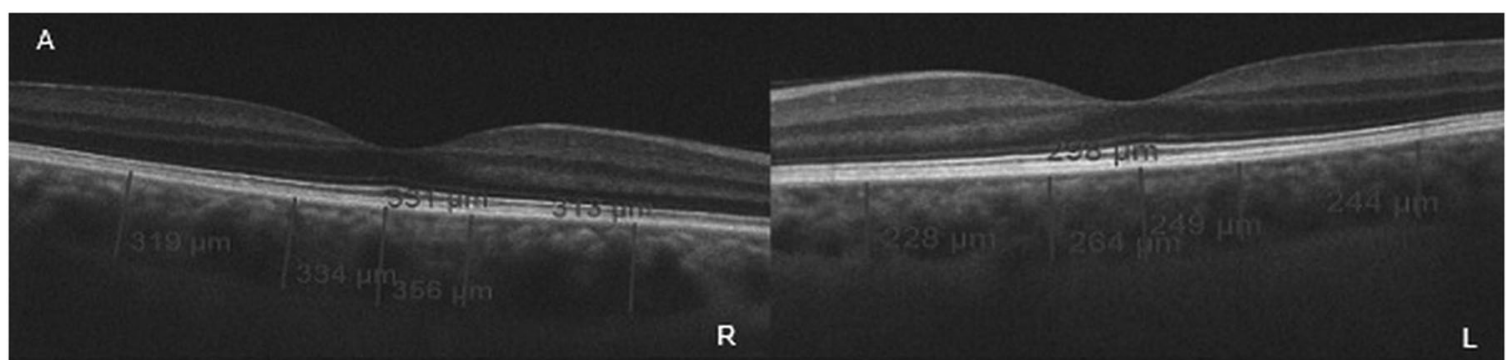

B

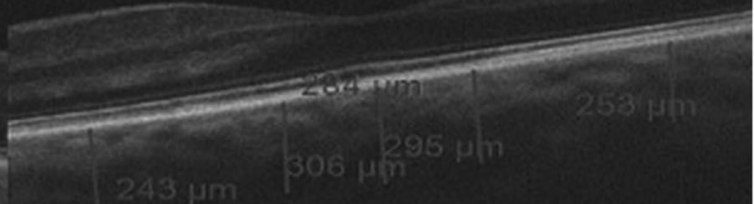

C

D

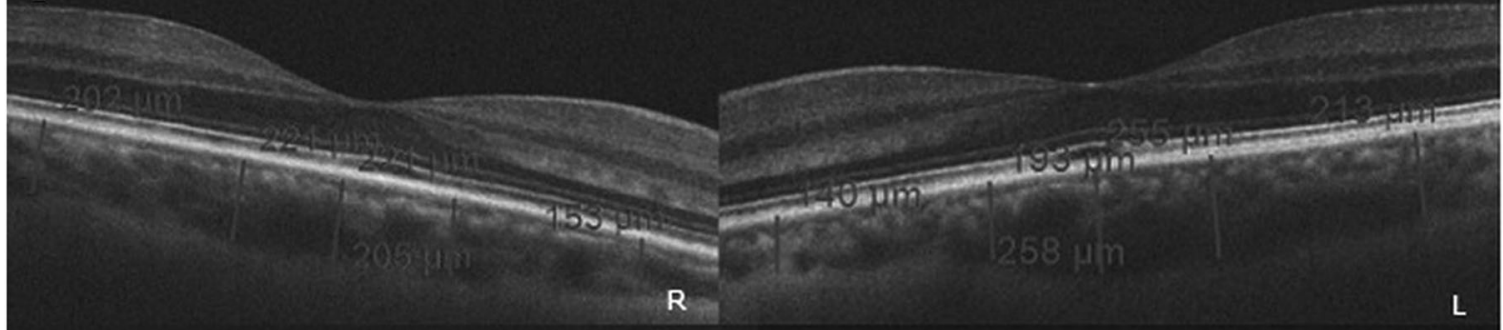

E

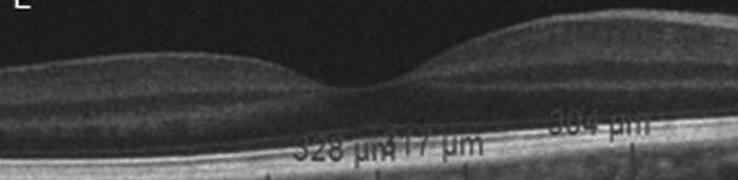

Fig. 1 a Measurements of choroidal thickness in the eyes of a patient with mild cardiovascular risk. $\mathbf{b}$ In the eyes of a patient with moderate cardiovascular risk. $\mathbf{c}$ In the eyes of a patient with high cardiovascular

\section{Discussion}

CAD is the leading cause of death, accounting for $25 \%$ of deaths in the United States and $31 \%$ worldwide [28]. Certain clinical studies declared that the Framingham Risk Score was not adequate to detect underlying cardiac disease risk. d In the eyes of a patient with very high cardiovascular risk. e In both eyes of healthy subjects.

in women due to atypical symptoms and coronary microvascular disease [29].

Various population-based studies reported significant associations between retinal microvascular changes and CAD. In large trials (ARIC, Blue Mountains Eye Study, and Beaver Dam Eye Study), a smaller arteriolar caliber and 
Table 1 Demographic and clinical characteristics of the study and control groups.

\begin{tabular}{lllllll}
\hline & Group 1 & Group 2 & Group 3 & Group 4 & Controls & $P$ \\
\hline Number of patients & 28 & 30 & 10 & 24 & 21 & \\
Age (years) & $40.1 \pm 6.5$ & $56.9 \pm 9.3$ & $68.0 \pm 5.2$ & $59.9 \pm 9.9$ & $48.8 \pm 5.9$ & 0.0001 \\
Gender (M/F) & $4 / 24$ & $7 / 23$ & $3 / 7$ & $17 / 7$ & $3 / 18$ & $0.0001^{*}$ \\
Smoking & 3 & 7 & 3 & 5 & 3 & 0.24 \\
Sistolic BP (mmHg) & $120 \pm 15.8$ & $130.3 \pm 16.9$ & $151 \pm 22.8$ & $129.1 \pm 12.8$ & $119.2 \pm 7.1$ & 0.0001 \\
Diastolic BP (mmHg) & $76.0 \pm 9.1$ & $79.9 \pm 11.5$ & $80 \pm 13.3$ & $77.7 \pm 8.5$ & $72.3 \pm 7.0$ & 0.081 \\
Cholesterol & $181.1 \pm 30.5$ & $227.1 \pm 40.1$ & $221.6 \pm 52.4$ & $191.7 \pm 52.6$ & $173.7 \pm 26.7$ & 0.0001 \\
LDL & $113.3 \pm 27.5$ & $149 \pm 33.7$ & $145.6+45.2$ & $119.6+44$ & $115.4+29.6$ & 0.0001 \\
HDL & $49+9.5$ & $53.9+11.8$ & $41.6+9.3$ & $41.8+9.1$ & $51.6+7.9$ & 0.0001 \\
\hline
\end{tabular}

$M$ Male, $F$ Female, BP Blood pressure, $L D L$ Low-density lipoprotein, HDL High-density lipoprotein. $* \chi^{2}$ test.

Table 2 Comparison of choroidal thickness measurements between healthy individuals and patients with cardiologic risk factors.

\begin{tabular}{|c|c|c|c|c|c|c|}
\hline & Group $1(n=28)$ & Group $2(n=30)$ & Group $3(n=10)$ & Group $4(n=24)$ & Controls $(n=21)$ & $P$ value \\
\hline $\mathrm{CMT}(\mathrm{R} / \mathrm{L}) \mu \mathrm{m}$ & $238.2 \pm 3.7$ & $240.6 \pm 20.9$ & $253.8 \pm 19.3$ & $258.4 \pm 34.3$ & $254.1 \pm 25.7$ & 0.057 \\
\hline Mean \pm SD & $238.6 \pm 30.2$ & $240.3 \pm 21$ & $249.4 \pm 25.6$ & $248.7 \pm 44.8$ & $252.6 \pm 27.2$ & 0.097 \\
\hline SFCT $(\mathrm{R} / \mathrm{L}) \mu \mathrm{m}$ & $311.21 \pm 77.7$ & $266.5 \pm 63.2$ & $264.7 \pm 57.5$ & $272.3 \pm 64.6$ & $352.0 \pm 74.4$ & 0.0001 \\
\hline Mean \pm SD & $303.5 \pm 49.6$ & $267.0 \pm 62.6$ & $272.3 \pm 64.6$ & $271.2 \pm 63.4$ & $363.1 \pm 89.0$ & 0.0001 \\
\hline $\mathrm{CT}[\mathrm{N}$ 0.5] (R/L) $\mu \mathrm{m}$ & $278.6 \pm 55.1$ & $248.3 \pm 58.1$ & $235.7 \pm 41.2$ & $243.2+55.1$ & $301.4+69.2$ & 0.002 \\
\hline Mean \pm SD & $276.7 \pm 44.5$ & $231.2 \pm 52.8$ & $238 \pm 58.2$ & $237.2+51.8$ & $296.2+85.6$ & 0.0001 \\
\hline $\mathrm{CT}[\mathrm{N} 1.5](\mathrm{R} / \mathrm{L}) \mu \mathrm{m}$ & $240 \pm 51.8$ & $205.7 \pm 57.8$ & $206 \pm 49.7$ & $210.8+65.3$ & $271.9+62.2$ & 0.001 \\
\hline Mean \pm SD & $247.2 \pm 47$ & $202.4 \pm 55.6$ & $210.8 \pm 50.1$ & $206.4+61.8$ & $262+69$ & 0.001 \\
\hline $\mathrm{CT}[\mathrm{T} \quad 0.5](\mathrm{R} / \mathrm{L}) \mu \mathrm{m}$ & $301.9 \pm 54.4$ & $251.1 \pm 58.9$ & $251.8 \pm 46.2$ & $262.2+60.3$ & $318+71.2$ & 0.0001 \\
\hline Mean \pm SD & $306.3 \pm 40.9$ & $253.8 \pm 52.1$ & $253.9 \pm 53.3$ & $263.7+52.4$ & $320.8+89.5$ & 0.0001 \\
\hline $\mathrm{CT}[\mathrm{T}$ 1.5] (R/L) $\mu \mathrm{m}$ & $262.6 \pm 58.5$ & $216.5 \pm 63.3$ & $223 \pm 49.1$ & $233.7+55.1$ & $272.1+62.2$ & 0.005 \\
\hline Mean \pm SD & $245.3 \pm 43.5$ & $216.9 \pm 51.3$ & $227.4 \pm 44.7$ & $220.4+52.9$ & $277.5+70$ & 0.001 \\
\hline
\end{tabular}

$C M T$ central macular thickness, $S F C T: C T: N(0.5)$ choroidal thickness at $500 \mu \mathrm{m}$ nasal to the fovea, $N(1.5)$ choroidal thickness at $1500 \mu \mathrm{m}$ nasal to the fovea, $T$ (0.5) choroidal thickness at $500 \mu \mathrm{m}$ temporal to the fovea, $T$ (1.5) choroidal thickness at $1500 \mu \mathrm{m}$ temporal to the fovea.

smaller arteriole to venule ratio correlated with a higher risk of coronary events in middle-aged women, but not in men or elderly subjects [30-32].

Wong et al. examined 6147 adults aged 45-84 years from the MESA cohort. They found a significant correlation between retinopathy and increased coronary artery calcium score when controlling for age, gender, race, and traditional risk factors. This relationship was not in accordance with changes in retinal microvascular caliber [33].

Although multiple clinical markers and risk factors have been defined, novel clinical markers and techniques are needed for evaluating risk stratification of CAD. The use of only retinal vasculature as a biomarker can lead to some difficulties with evaluating CAD [28]. On the other hand, the ability to explore the choroid layers using EDI SD-OCT provides new options to research patients with CVRF and patients with CAD. In this study, we investigated whether the choroid layers may be used as a novel biomarker in the diagnosis and early management of patients with CAD.
The choroid, a highly vascularized tissue, provides oxygen and nourishment to the outer retinal layers and regulates temperature at the macular area. Choroidal thickness and ocular perfusion pressure in healthy subjects are related, and SFCT is considered as an indirect index of ocular perfusion status [31].

$\mathrm{CT}$ is well-known to be affected by various systemic and ocular factors. Gender and age are linked with CT differences; men have higher CT than women due to hormonal factors and sympathetic tone [7]. In the current study, the CAD group was the only one that demonstrated a significant difference in age or gender distribution.

Although various studies reported increased choroidal thickness in patients with diabetes mellitus, the results seem to be debatable $[16,30,31]$. The consensus is that diabetes results in a decrement of choroidal depth. For this reason, we did not recruit diabetic patients for our study.

In a cross-sectional observational study of 322 healthy eyes of 161 subjects (mean age 60 years), Wong et al. found 
the subfoveal choroidal thickness was significantly higher in subjects with hypercholesterolemia $(306 \pm 111 \mathrm{~mm})$ as compared with controls $(258 \pm 97 \mathrm{~mm})$. Wong et al. speculated that hypercholesterolemia might be a crucial factor that should be taken into consideration in CT [11]. In the present study, LDL levels were also found to be inversely correlated with SFCT in both eyes of the study groups, but there was no significant correlation between cholesterol levels and macular CT.

Smoking, which is one of the most important modifiable risk factors for many cardiovascular, respiratory, and other systemic diseases [28], decreases blood circulation in retinal and choroid layers $[34,35]$. A significant decrease in choroidal thickness has been found in certain studies that are related to reduced nitric oxide bioavailability [36, 37]. NO causes peripheral vasoconstriction and thereby reduces CT. According to our research, no significant correlation exists between smoking and choroidal thickness. Each group had a low number of smokers that may have caused this statistical result.

Hypertension induces pathology in vascular systems, including the heart, brain, kidneys, and eyes. Ocular changes resulting from hypertension include retinal hemorrhages, cotton wool spots, intraretinal lipid accumulation, and vessel closure in the retinal capillaries and the choriocapillaris. Although both retinal and choroidal changes are observed with hypertension, the mechanism of choroidal deterioration remains obscure. Ahn et al., based on 42 eyes with hypertensive retinopathy, demonstrated a significant increase in subfoveal choroidal thickness, likely due to interstitial fluid accumulation in the choroid caused by choroidal permeability changes [8]. Gok et al., based on 228 eyes of 116 patients with chronic hypertensive, reported no significant difference in subfoveal choroidal thickness between the study group and the controls. Gok et al. also emphasized that neither arterial stiffness nor left ventricular hypertrophy appears to be associated with any change to the SFCT in patients with essential chronic hypertension [14]. It seems to be related to the protection of the choroidal vascular network from the adverse effects of chronic hypertension. In our study, we also detected no significant correlation between systolic blood pressure and CT but found a positive relationship between systolic blood pressure and the SCORE System in all patients [27].

Altinkaynak et al. investigated CT by comparing 56 eyes of 56 patients with chronic heart failure (CHF) to 56 eyes of 56 gender-matched individuals and found that the mean SFCT value was statistically significantly lower in patients with CHF. Altinkaynak et al. proposed two possible mechanisms: low cardiac output may result in the development of vasoconstriction in the orbital and choroidal vessels; second, vasoconstriction results in chronic ischemia and RPE atrophy [13]. Ahma et al. evaluated CT by comparing 34 eyes of 34 patients with CAD to 28 eyes of 28 age-matched controls and reported a thinner macular choroid in CAD groups as compared with controls [38].

In our study, all study groups had a lower mean SFCT than controls for both eyes, and these differences were statistically significant. At each nasal and temporal region of the macula, the mean CT values of both eyes of patients with CVRF (in the moderate-risk group and high-risk group) and the very high-risk group (patients with $\mathrm{CAD}$ ) were significantly decreased when compared with controls.

Certain layers, such as the ellipsoid zone and outer retina, are supplied by choroidal circulation. Photoreceptors require most of the oxygen from the choroid. After having adjusted patients for age, gender, and race, at baseline, Duan et al. showed in their study that patients with MI who were more likely to develop choroidal neovascularization with a $\% 26$ percentage [39]. In light of these findings, choroidal ischemia may be related to cardiogenic ischemia and cardiogenic risk factors.

Our study has several limitations. The study groups were relatively small. We found only a low number of patients with high cardiovascular risk (Group 3). Patients with high myopia were excluded from the study groups; we did not collect quantitative axial length data and therefore, cannot account for variations in CT due to myopia of less than 3D and thus cannot rule out possible smaller effects of axial length on CT.

Strengths of the study include the identification of wellcharacterized, prospectively recruited subjects with CVRF from cardiology clinics, carefully selected, age-matched controls, and exclusion of diabetic patients. Poor-quality imaging data were omitted. The differences in CT between the groups were highly remarkable, and these differences were found at five measurement points in the macula.

In conclusion, SDD and reticular pseudodrusen (RPD) has been shown to be related to choroidal thinning, which has been reported in patients with CAD and various systemic vascular disease. SDD is classified into three stages of severity, and this staging was used by Cymerman et al. to study SDD and CAD (24). In our study, the rate of SDD was also higher in patients with CVRF as compared with controls, and the rate and severity both increased in accordance with the severity of CAD, a stronger finding than that just cited. We evaluated $\mathrm{CT}$ in study groups that were compared with age-matched controls and found an independent, negative association between CT and CVRF. This negative association was significant at the temporal and nasal regions of the retina in patients with CVRF (moderate risk, high risk, and very high risk). These findings suggest that CT may represent a promising and reassuring disease marker before the development of CAD that may be able to provide crucial information regarding both systemic cardiovascular health and susceptibility to disease of the outer 
retina, RPE, and choroid. Further clinical trials are required to define the effect of CVRF on the retina and choroid with EDI-OCT or swept-source OCT in larger series with longer follow-up.

\section{Summary}

\section{What was known before}

- Coronary heart disease (CAD) is a major cause of death in the middle-aged people.

- Computerized techniques have been developed to measure the retinal a vessel via retinal imaging has enabled to speculate increased risk of CAD in epidemiological studies.

- Choroid may be prone to arteriosclerotic processes as the other organs and affected by systemic factors such as cigarette smoking, ocular ischemic syndrome, chronic heart failure, and systemic hypertension, carotid artery stenosis, diabetes mellitus.

\section{What this study adds}

- No previous studies have investigated association between measurements of central macular thickness (CMT), choroidal thickness (CT) and cardiovascular risk factors (CVRF).

- Our study aimed to examine CMT and CT in eyes of patients with CVRF or coronary heart disease (CAD).

- EDI-OCT is a rapid, non-invasive, high-resolution procedure with which to monitor the choroid features. We consider that it may be used as a promising and reassuring disease marker prior to the development of $\mathrm{CAD}$ in the future.

\section{Compliance with ethical standards}

Conflict of interest The authors declare that they have no conflict of interest.

Publisher's note Springer Nature remains neutral with regard to jurisdictional claims in published maps and institutional affiliations.

\section{References}

1. Dzau VJ, Antman EM, Black HR, Hayes DL, Manson JE, Plutzky J, et al. The cardiovascular disease continuum validated: clinical evidence of improved patient outcomes: part I: pathophysiology and clinical trial evidence (risk factors through stable coronary artery disease). Circulation. 2006;114:2850-70.

2. Grundy SM, Cleeman JI, Daniels SR, Donato KA, Eckel RH, Franklin BA, et al. Diagnosis and management of the metabolic syndrome: an American Heart Association/National Heart, Lung, and Blood Institute Scientific Statement. Circulation. 2005; 112:2735-52.

3. Pearson TA, Blair SN, Daniels SR, Eckel RH, Fair JM, Fortmann SP, et al. AHA guidelines for primary prevention of cardiovascular disease and stroke: 2002 update: consensus panel guide to comprehensive risk reduction for adult patients without coronary or other atherosclerotic vascular diseases. American Heart Association Science Advisory and Coordinating Committee. Circulation. 2002;106:388-91.

4. Tanaka M, Fujiwara H, Onodera T, Wu DJ, Matsuda M, Hamashima Y, et al. Quantitative analysis of narrowings of intramyocardial small arteries in normal hearts, hypertensive hearts, and hearts with hypertrophic cardiomyopathy. Circulation. 1987; 75:1130-9.

5. McGeechan K, Liew G, Macaskill P, Irwig L, Klein R, Klein BE, et al. Meta-analysis: retinal vessel caliber and risk for coronary heart disease. Ann Intern Med. 2009;151:404-13.

6. Spaide RF, Koizumi H, Pozzoni MC. Enhanced depth imaging spectral-domain optical coherence tomography. Am J Ophthalmol. 2008;146:496-500.

7. Karapetyan A, Ouyang P, Tang LS, Gemilyan M. Choroidal thickness in relation to ethnicity measured using enhanced depth imaging optical coherence tomography. Retina. 2016;36:82-90. https://doi.org/10.1097/IAE.0000000000000654.

8. Branchini LA, Adhi M, Regatieri CV, Nandakumar N, Liu JJ, Laver N, et al. Analysis of choroidal morphologic features and vasculature in healthy eyes using spectral-domain optical coherence tomography. Ophthalmology. 2013;120:1901-8.

9. Tan KA, Gupta P, Agarwal A, Chhablani J, Cheng CY, Keane PA, et al. State of science: Choroidal thickness and systemic health. Surv Ophthalmol. 2016;61:566-81.

10. Ahn SJ, Woo SJ, Park KH. Retinal and choroidal changes with severe hypertension and their association with visual outcome. Invest Ophthalmol Vis Sci. 2014;55:7775-85.

11. Wong IY, Wong RL, Zhao P, Lai WW. Choroidal thickness in relation to hypercholesterolemia on enhanced depth imaging optical coherence tomography. Retina. 2013;33:423-8.

12. Kim DY, Joe SG, Lee JY, Kim JG, Yang SJ. Choroidal thickness in eyes with unilateral ocular ischemic syndrome. J Ophthalmol. 2015;2015:620372.

13. Altinkaynak H, Kara N, Sayin N, Gunes H, Avsar S, Yazici AT. Subfoveal choroidal thickness in patients with chronic heart failure analyzed by spectral-domain optical coherence tomography. Curr Eye Res. 2014;39:1123-8.

14. Gok M, Karabas VL, Emre E, Aksar AT, Aslan MS, Ural D. Evaluation of choroidal thickness via enhanced depth-imaging optical coherence tomography in patients with systemic hypertension. Indian J Ophthalmol. 2015;63:239-43.

15. Sayin N, Kara N, Uzun F, Akturk IF. A quantitative evaluation of the posterior segment of the eye using spectral-domain optical coherence tomography in carotid artery stenosis: a pilot study. Ophthalmic Surg Lasers Imaging Retin. 2015;46:180-5. https:// doi.org/10.3928/23258160-20150213-20.

16. Xu J, Xu L, Du KF, Shao L, Chen CX, Zhou JQ, et al. Subfoveal choroidal thickness in diabetes and diabetic retinopathy. Ophthalmology. 2013;120:2023-8.

17. Klaver CC, Wolfs RC, Vingerling JR, Hofman A, de Jong PT. Age-specific prevalence and causes of blindness and visual impairment in an older population: the Rotterdam Study. Arch Ophthalmol. 1998;116:653-8.

18. Smith RT, Sohrab MA, Busuioc M, Barile G. Reticular macular disease. Am J Ophthalmol. 2009;148:733-43.e732.

19. Rudolf M, Malek G, Messinger JD, Clark ME, Wang L, Curcio CA. Sub-retinal drusenoid deposits in human retina: organization and composition. Exp Eye Res. 2008;87:402-8. 
20. Pumariega NM, Smith RT, Sohrab MA, Letien V, Souied EH. A prospective study of reticular macular disease. Ophthalmology. 2011;118:1619-25.

21. Xu L, Blonska AM, Pumariega NM, Bearelly S, Sohrab MA, Hageman GS, et al. Reticular macular disease is associated with multilobular geographic atrophy in age-related macular degeneration. Retina. 2013;33:1850-62.

22. Alten F, Heiduschka P, Clemens CR, Eter N. Exploring choriocapillaris under reticular pseudodrusen using OCTAngiography. Graefes Arch Clin Exp Ophthalmol. 2016; 254:2165-73.

23. Lains I, Wang J, Providencia J, Mach S, Gil P, Gil J, et al. Choroidal changes associated with subretinal drusenoid deposits in age-related macular degeneration using swept-source optical coherence tomography. Am J Ophthalmol. 2017;180:55-63.

24. Cymerman RM, Skolnick AH, Cole WJ, Nabati C, Curcio CA, Smith RT. Coronary artery disease and reticular macular disease, a subphenotype of early age-related macular degeneration. Curr Eye Res. 2016;41:1482-8.

25. Leisy HB, Ahmad M, Marmor M, Smith RT. Association between decreased renal function and reticular macular disease in agerelated macular degeneration. Ophthalmol Retin. 2017;1:42-8.

26. Smith RT, Merriam JE, Sohrab MA, Pumariega NM, Barile G, Blonska AM, et al. Complement factor $\mathrm{H} 402 \mathrm{H}$ variant and reticular macular disease. Arch Ophthalmol. 2011;129:1061-6.

27. Piepoli MF, Hoes AW, Agewall S, Albus C, Brotons C, Catapano AL, et al. 2016 European guidelines on cardiovascular disease prevention in clinical practice. The Sixth Joint Task Force of the European Society of Cardiology and Other Societies on Cardiovascular Disease Prevention in Clinical Practice (constituted by representatives of 10 societies and by invited experts. Developed with the special contribution of the European Association for Cardiovascular Prevention \& Rehabilitation. G Ital Cardiol (Rome). 2017;18:547-612.

28. WHO. Cardiovascular diseases (CVDs) fact sheet. (September 2016. http://www.who.int/mediacentre/factsheets/fs317/en/. Cited 13 May 2016).

29. Reis SE, Holubkov R, Conrad Smith AJ, Kelsey SF, Sharaf BL, Reichek N, et al. Coronary microvascular dysfunction is highly prevalent in women with chest pain in the absence of coronary artery disease: results from the NHLBI WISE study. Am Heart J. 2001;141:735-41.

30. Wang JJ, Liew G, Wong TY, Smith W, Klein R, Leeder SR, et al. Retinal vascular calibre and the risk of coronary heart diseaserelated death. Heart. 2006;92:1583-7.

31. Wong TY, Klein R, Nieto FJ, Klein BE, Sharrett AR, Meuer SM, et al. Retinal microvascular abnormalities and 10-year cardiovascular mortality: a population-based case-control study. Ophthalmology. 2003;110:933-40.

32. Wong TY, Klein R, Sharrett AR, Duncan BB, Couper DJ, Tielsch $\mathrm{JM}$, et al. Retinal arteriolar narrowing and risk of coronary heart disease in men and women. The atherosclerosis risk in communities study. JAMA. 2002;287:1153-9.

33. Wong TY, Cheung N, Islam FM, Klein R, Criqui MH, Cotch MF, et al. Relation of retinopathy to coronary artery calcification: the multi-ethnic study of atherosclerosis. Am J Epidemiol. 2008; 167:51-8.

34. Langhans M, Michelson G, Groh MJ. Effect of breathing 100\% oxygen on retinal and optic nerve head capillary blood flow in smokers and non-smokers. Br J Ophthalmol. 1997;81:365-9.

35. Wimpissinger B, Resch H, Berisha F, Weigert G, Schmetterer L, Polak K. Response of choroidal blood flow to carbogen breathing in smokers and non-smokers. Br J Ophthalmol. 2004; 88:776-81.

36. Sizmaz S, Kucukerdonmez C, Pinarci EY, Karalezli A, Canan H, Yilmaz G. The effect of smoking on choroidal thickness measured by optical coherence tomography. $\mathrm{Br} \mathrm{J}$ Ophthalmol. 2013; 97:601-4.

37. Ulas F, Celik F, Dogan U, Celebi S. Effect of smoking on choroidal thickness in healthy smokers. Curr Eye Res. 2014;39:504-11. https://doi.org/10.3109/02713683.2013.850099.

38. Ahmad M, Kaszubski PA, Cobbs L, Reynolds H, Smith RT. Choroidal thickness in patients with coronary artery disease. PLoS ONE. 2017;12:e0175691.

39. Duan Y, Mo J, Klein R, Scott IU, Lin HM, Caulfield vne, et al. Age-related macular degeneration is associated with incident myocardial infarction among elderly Americans. Ophthalmology. 2007;114:732-7. 